\title{
Ergonomic Injuries in Endoscopists and Their Risk Factors
}

\author{
Lubna Kamani ${ }^{1}$ and Hamid Kalwar ${ }^{2}$ \\ ${ }^{1}$ Department of Gastroenterology, Liaquat National Hospital, Karachi, ${ }^{2}$ Murshid Hospital, Karachi, Pakistan
}

Background/Aims: Prolonged repetitive strain caused by the continuous performance of complex endoscopic procedures enhances the risk of ergonomic injuries among health-care providers (HCPs), specifically endoscopists. This study aimed to assess the risk factors of ergonomic injuries among endoscopists and non-endoscopists.

Methods: This cross-sectional study was conducted at the Gastroenterology Department of Liaquat National Hospital, Karachi, Pakistan. A total of 92 HCPs were enrolled, of whom 61 were involved in endoscopic procedures and 31 were non-endoscopists. Data were collected through a self-administered questionnaire during national gastroenterology conferences and analyzed using SPSS version 22 (IBM Corp. Chicago, IL, USA).

Results: Of the total study population, $95.08 \%$ of endoscopists were observed to have ergonomic injuries, whereas only $54.83 \%$ of non-endoscopists had ergonomic injuries $(p<0.00)$. The most common injury associated with musculoskeletal (MSK) pain sites was back (41\%), leg (23\%), and hand (19.7\%) pain among endoscopists. Of 28 endoscopists performing $\geq 20$ procedures/week, 26 had MSK injury. However, $95.08 \%$ of endoscopists had developed MSK injury irrespective of working hours ( $>5 \mathrm{or}<5 \mathrm{hr} / \mathrm{wk})$.

Conclusions: Endoscopists are at high risk of developing ergonomic injuries, representing the negative potential of the endoscopyassociated workload. To overcome these issues, an appropriate strategic framework needs to be designed to avoid occupational compromises. Clin Endosc 2021;54:356-362

Key Words: Endoscopy; Ergonomics; Health-care providers; Musculoskeletal injury; Occupational performance

\section{INTRODUCTION}

Work-related musculoskeletal (MSK) injury is the principal occupational health problem reported by the majority of health-care providers (HCPs), as routine practice poses a continuous risk. Moreover, MSK injury is an often reported complaint among endoscopists. ${ }^{1-5}$ The continuous occupational load and its associated risks have been known to affect the overall quality of life and the working routine of physicians. Ergonomic injuries have been reported to occur owing to stat-

Received: July 28, 2020 Revised: November 28, 2020

Accepted: December 1, 2020

Correspondence: Lubna Kamani

Department of Gastroenterology, Liaquat National Hospital, National Stadium Road, Karachi 74800, Pakistan.

Tel: +92-30-02562141, Fax: +92-21-34140014, E-mail: lkamani@yahoo.com ORCID: https://orcid.org/0000-0003-2651-5179

(cc) This is an Open Access article distributed under the terms of the Creative Commons Attribution Non-Commercial License (http://creativecommons.org/ licenses/by-nc/3.0) which permits unrestricted non-commercial use, distribution, and reproduction in any medium, provided the original work is properly cited. ic muscle loading, repetitive movements, and inappropriate body posture during procedures. ${ }^{6,7}$ Additionally, continuous repetitive procedures, lack of breaks, and personal routines and practices of HCPs double the risk of occupational injuries. $^{8}$

Ergonomic injuries leading to pain in MSK sites (neck, low back, thumb, and hand) are frequently reported by HCPs, specifically endoscopists. ${ }^{3,4}$ Previous studies have reported that the overall prevalence of these procedural injuries among endoscopists ranges from $39 \%$ to $89 \% .{ }^{9}, 10$ Endoscopy has become the most important and frequently performed procedure in gastroenterology. ${ }^{11}$ Therefore, the injury risk is comparatively higher among physicians and surgeons involved in endoscopic procedures. Additionally, the disease crisis has intensified during the recent years, thus increasing procedural stress and predisposing endoscopists to occupational injuries at a high rate. $^{12}$

Currently, work-related MSK disorders and the associated ergonomic mechanisms are points of concern for the health sector. The condition is threatening for both HCPs and patients, as it results in compromised occupational per- 
formance. ${ }^{13}$ MSK complaints are known to interfere with the normal work routine among endoscopists. In a previous study, approximately $84.6 \%$ endoscopists had MSK pain, which greatly affected their work routine. ${ }^{14}$

The demand for endoscopic procedures is expected to further increase over time. ${ }^{12,15}$ Therefore, it is crucial to explore and address the ergonomic aspects of endoscopy. Although alarming outcomes have been previously reported in several international studies, very little is known about the health issues faced by most endoscopists worldwide. The basic objective of this study was to estimate the prevalence of ergonomic injuries and associated MSK pain among endoscopists in comparison with non-endoscopists. We sought to assess the risk factors and characteristics related to routine practice that contribute to the development of these ergonomic injuries.

\section{MATERIALS AND METHODS}

A cross-sectional study was planned at a tertiary care hospital (Liaquat National Hospital [LNH]) in Karachi, Pakistan. Data were collected during a period of 6 months (August 2019 to January 2020). A total of $92 \mathrm{HCPs}$ were enrolled in the study, including 61 physicians and surgeons involved in endoscopic procedures and 31 non-endoscopist physicians. The data were collected during national gastroenterology conferences across Pakistan. The study questionnaire was completed on a voluntary basis.

The study was designed according to the Declaration of Helsinki, and ethical approval was obtained from the institutional review board of LNH. Written informed consent was obtained from each HCP before enrollment in the study.

A self-administered questionnaire was designed for endoscopists, inquiring demographic details such as age, sex, weight, height, body mass index (BMI), comorbid conditions, and gastroenterology practice characteristics, such as preferred glove size and gastroenterology training. Moreover, ergonomic injuries and associated pain, including thumb, shoulder, hand, neck, back, leg, and wrist pain, were assessed in detail. Risk factors influencing ergonomic injuries and MSK pain, such as hand dominance, number of procedures performed per week, time spent performing endoscopy, participation in exercises, and cumulative duration of performing endoscopy, were also noted. However, a modified questionnaire omitting endoscopy-associated characteristics was used for non-endoscopist physicians.

The recorded data were analyzed using SPSS version 22 (IBM Corp. Chicago, IL, USA). All continuous variables, including age, weight, height, and BMI, are presented as mean \pm standard deviation. Qualitative variables, including age group, comorbidities, glove size, training, breaks, hand dominance, and various other characteristics associated with the practice of endoscopists, are presented as $n(\%)$. Student's $t$-test was performed to evaluate differences in weight, height, and BMI between the groups, whereas the chi-square test was used to compare categorical variables. To identify the factors associated with MSK pain among endoscopists, univariate logistic regression analysis was performed, and a $p$-value of $<0.05$ was considered significant.

\section{RESULTS}

Responses were received from 61 endoscopists and 31 non-endoscopists. The details of the baseline characteristics of the two groups are provided in Table 1. Similar trends were observed between groups in terms of age, height, BMI, hand dominance, and sex. Both endoscopists and non-endoscopists were engaged in exercise. The overall frequency of MSK pain varied between the two groups $(p<0.05)$. Collectively, the injuries and MSK pain were found to be associated with performing endoscopic procedures. Approximately $95.08 \%$ of endoscopists reported having MSK pain, whereas only 54.83\% of non-endoscopists complained of MSK pain. Furthermore, most of the endoscopists with these injuries were overweight or obese (68.8\%).

\section{Incidence of musculoskeletal injury according to details of endoscopic procedures}

According to the reported data, higher procedural rate, increased procedural duration, and lack of breaks during the procedures influenced the overall incidence of MSK injuries among endoscopists. Approximately $44.8 \%$ of endoscopists performing $\geq 20$ procedures/week had an MSK injury. Moreover, $86.2 \%$ of those with MSK injuries spent up to $5 \mathrm{hr} /$ week performing endoscopy, whereas $13.7 \%$ spent $6-10 \mathrm{hr} /$ week performing endoscopic procedures. The most performed procedures by the endoscopists with MSK injury were gastroscopy and colonoscopy (44.8\%). Furthermore, 26 (44.8\%) endoscopists did not take regular breaks during the procedures and were found to have an MSK injury (Table 2).

\section{Musculoskeletal pain among endoscopists and non- endoscopists}

As shown in Fig. 1, the complaints about endoscopy-related MSK pain were mostly associated with lower back pain (40.9\%), followed by leg pain (24.5\%). Pain in the hand, thumb, wrist, and neck was reported by $22.9 \%, 21.3 \%, 13.1 \%$, 
Table 1. Baseline Characteristics of the Study Population

\begin{tabular}{|c|c|c|c|c|}
\hline Variable & & Endoscopists $(n=61) N(\%)$ & Non-endoscopists $(n=31) N(\%)$ & $p$-value \\
\hline Age $(y r)$, mean $\pm S D$ & & $44.02 \pm 7.8$ & $42.39 \pm 8.7$ & 0.003 \\
\hline \multirow[t]{2}{*}{ Gender } & Male & $58(95.08)$ & $12(38.7)$ & 0.000 \\
\hline & Female & $3(4.91)$ & $19(61.29)$ & \\
\hline Weight $(\mathrm{kg})$, mean $\pm \mathrm{SD}$ & & $80 \pm 12.7$ & $65.5 \pm 9.9$ & 0.000 \\
\hline Height $(m)$, mean \pm SD & & $1.76 \pm 0.12$ & $1.67 \pm 0.13$ & 0.822 \\
\hline BMI $\left(\mathrm{kg} / \mathrm{m}^{2}\right)$, mean $\pm \mathrm{SD}$ & & $25.53 \pm 4.25$ & $23.30 \pm 2.86$ & 0.148 \\
\hline \multirow[t]{4}{*}{ BMI groups } & Underweight & $1(1.63)$ & - & 0.217 \\
\hline & Normal & $15(24.59)$ & $3(9.67)$ & \\
\hline & Overweight & $17(27.86)$ & $13(41.93)$ & \\
\hline & Obese & $28(45.90)$ & $15(48.38)$ & \\
\hline \multirow[t]{2}{*}{ Age group } & $<50 \mathrm{yr}$ & $49(80.32)$ & $25(80.64)$ & 0.971 \\
\hline & $>50 \mathrm{yr}$ & $12(19.67)$ & $6(19.35)$ & \\
\hline \multirow[t]{4}{*}{ Comorbidities } & Asthma & $2(3.3)$ & - & 0.063 \\
\hline & Hypertension & $11(18.03)$ & $1(3.2)$ & \\
\hline & Diabetes mellitus & - & $2(6.5)$ & \\
\hline & None & $48(78.7)$ & $28(90.3)$ & \\
\hline \multirow[t]{2}{*}{ Regular medication } & Yes & $12(19.7)$ & $7(22.6)$ & 0.000 \\
\hline & No & $49(80.3)$ & $24(77.4)$ & \\
\hline \multirow[t]{5}{*}{ Medication for comorbidities } & Anti-hypertension & $5(8.2)$ & - & - \\
\hline & $\mathrm{ARB}$ & $3(4.9)$ & - & \\
\hline & Thyroxin & $1(1.6)$ & - & \\
\hline & None & $49(80.3)$ & - & \\
\hline & No response & $2(3.27)$ & - & \\
\hline \multirow[t]{2}{*}{ Training attained } & Yes & $59(96.72)$ & $31(100)$ & 0.000 \\
\hline & No & $2(3.27)$ & - & \\
\hline \multirow[t]{4}{*}{ Training years } & $2-3 \mathrm{yr}$ & $36(59.01)$ & - & 0.000 \\
\hline & $4-6 \mathrm{yr}$ & $22(36.06)$ & $31(100)$ & \\
\hline & $8 \mathrm{yr}$ & $1(1.63)$ & - & \\
\hline & No & $2(3.27)$ & - & \\
\hline \multirow[t]{2}{*}{ Hand dominance } & Left hand & $5(8.19)$ & $2(6.45)$ & 0.765 \\
\hline & Right hand & $56(91.80)$ & $29(93.54)$ & \\
\hline \multirow[t]{2}{*}{ Musculoskeletal injury } & Present & $58(95.08)$ & $17(54.83)$ & 0.000 \\
\hline & Absent & $3(4.91)$ & $14(22.95)$ & \\
\hline
\end{tabular}

$\mathrm{ARB}$, angiotensin II receptor blockers; BMI, body mass index; SD, standard deviation.

${ }^{*} p$-value $<0.05$ is considered significant. 
Table 2. Incidence of Musculoskeletal Injury according to Endoscopy Workload Associated Details

\begin{tabular}{|c|c|c|c|c|}
\hline \multirow{2}{*}{ Procedure details } & & \multicolumn{2}{|c|}{ Musculoskeletal injury } & \multirow{2}{*}{$p$-value } \\
\hline & & Present $(n=58) N(\%)$ & Absent $(n=3) N(\%)$ & \\
\hline \multirow[t]{2}{*}{ Endoscopies performed/week } & $\geq 20$ procedures & $26(44.8)$ & $2(66.6)$ & 0.718 \\
\hline & $<20$ procedures & $32(55.2)$ & $1(33.3)$ & \\
\hline \multirow{2}{*}{$\begin{array}{l}\text { Time spent performing } \\
\text { endoscopy }\end{array}$} & $\leq 5 \mathrm{hr} / \mathrm{wk}$ & $50(86.2)$ & $3(100)$ & 0.490 \\
\hline & $>5 \mathrm{hr} / \mathrm{wk}$ & $8(13.7)$ & - & \\
\hline \multirow{2}{*}{$\begin{array}{l}\text { Cumulative years performing } \\
\text { endoscopy }\end{array}$} & $\leq 20 \mathrm{yr}$ & $55(94.8)$ & $3(100)$ & 0.344 \\
\hline & $>20 \mathrm{yr}$ & $3(5.17)$ & - & \\
\hline \multirow[t]{2}{*}{ Procedures with ERCP \& EUS } & Yes & $31(53.4)$ & $1(33.3)$ & 0.496 \\
\hline & No & $27(46.6)$ & $2(66.7)$ & \\
\hline \multirow[t]{4}{*}{ Procedures type } & Colonoscopy & $1(1.7)$ & $1(33.3)$ & $0.026^{*}$ \\
\hline & Gastroscopy and colonoscopy & $26(44.8)$ & $1(33.3)$ & \\
\hline & Gastroscopy and colonoscopy, ERCP & $23(39.6)$ & $1(33.3)$ & \\
\hline & Gastroscopy and colonoscopy, ERCP, EUS & $8(13.7)$ & - & \\
\hline \multirow[t]{5}{*}{ Preferred glove size } & 6.5 & $1(1.7)$ & - & 0.884 \\
\hline & 7.0 & $14(24.1)$ & - & \\
\hline & 7.5 & $30(51.7)$ & $2(66.6)$ & \\
\hline & 8.0 & $12(20.7)$ & $1(33.3)$ & \\
\hline & 8.5 & $1(1.7)$ & - & \\
\hline \multirow{2}{*}{$\begin{array}{l}\text { Regular breaks between } \\
\text { procedure }\end{array}$} & Yes & $32(55.1)$ & $2(66.6)$ & 0.696 \\
\hline & No & $26(44.8)$ & $1(33.3)$ & \\
\hline \multirow{2}{*}{$\begin{array}{l}\text { Duration of regular breaks } \\
\text { between procedures }\end{array}$} & $0-10 \mathrm{~min}$ & $31(53.4)$ & $2(66.6)$ & 0.979 \\
\hline & $21-30 \mathrm{~min}$ & $1(1.72)$ & - & \\
\hline
\end{tabular}

ERCP, endoscopic retrograde cholangiopancreatography; EUS, endoscopic ultrasound.

${ }^{*} p$-value $<0.05$ is considered significant.

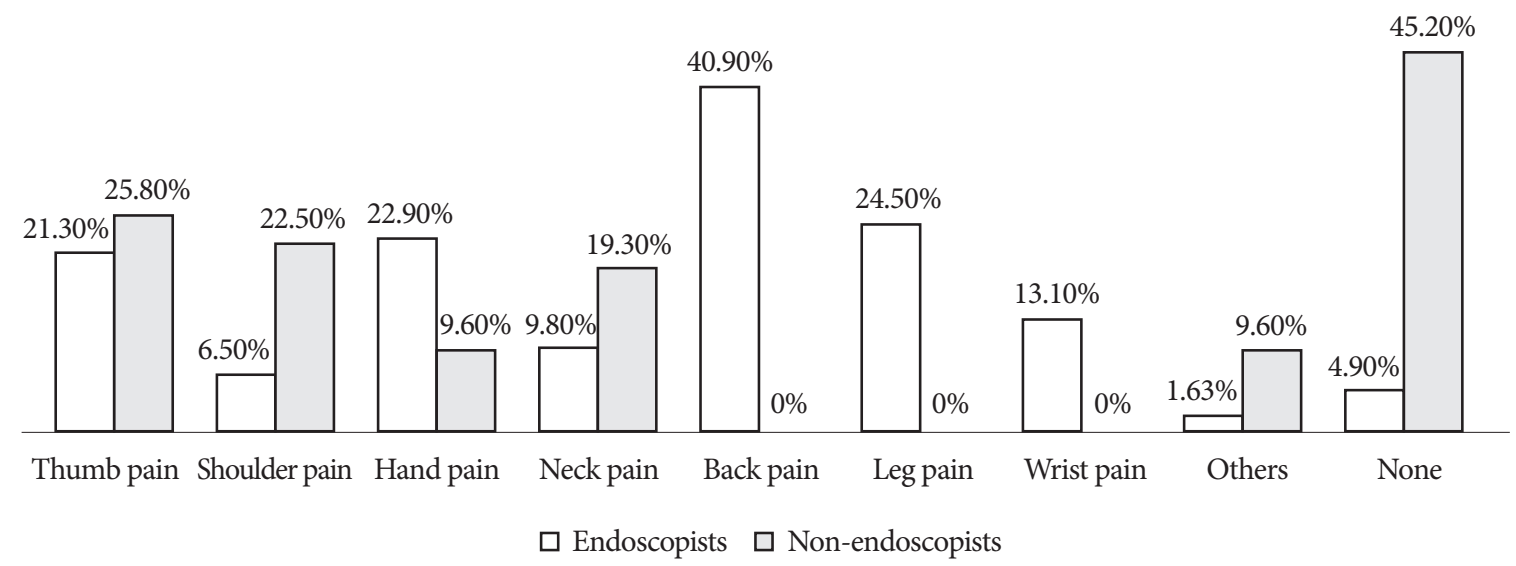

Fig. 1. Site of musculoskeletal pain among endoscopists and non-endoscopists. 


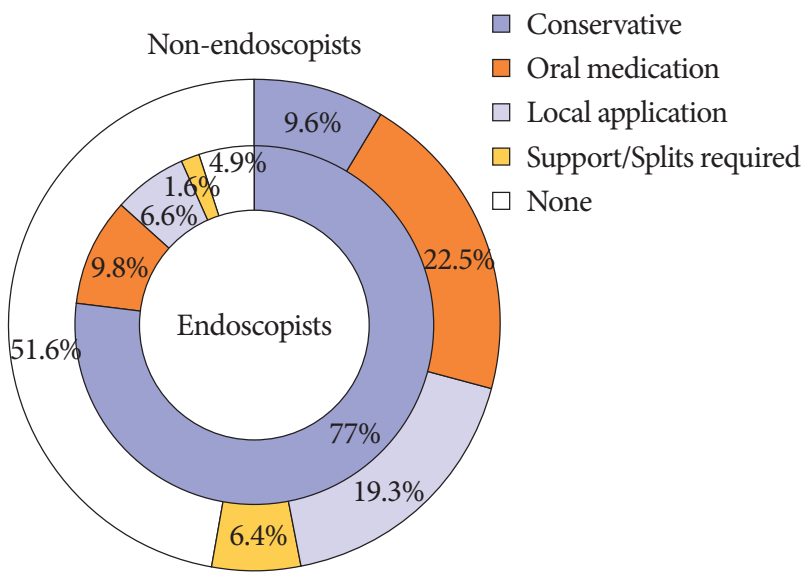

Fig. 2. Treatments preferred by endoscopists and non-endoscopists for ergonomic injuries and musculoskeletal pain.

and $9.8 \%$, respectively. The most common sites of pain among non-endoscopists were the thumb (25.8\%), shoulder (22.5\%), and neck (19.3\%).

Oral medication was the preferred treatment (22.5\%) for ergonomic injuries and MSK pain among the non-endoscopists, followed by locally applied treatment (19.3\%), whereas $51.6 \%$ preferred no treatment. In contrast, $77 \%$ of the endoscopists preferred conservative treatment, $9.8 \%$ preferred oral medication, $6.6 \%$ preferred locally applied treatment, and $4.9 \%$ preferred none of these treatments for ergonomic injuries and associated MSK pain (Fig. 2).

\section{Factors associated with ergonomic injuries and musculoskeletal pain among endoscopists}

The results of univariate analysis for factors related to ergonomic injuries and MSK pain among the enrolled endoscopists are provided in Table 3. None of the predictors were significantly associated with MSK injuries ( $p>0.05)$. A non-significant difference was observed with respect to procedural frequency and susceptibility to ergonomic injuries: endoscopists performing $\geq 20$ procedures/week were only 1.082 times more likely to develop ergonomic injuries than those performing $<20$ procedures/week. Those who were taking regular breaks between the procedures had a 0.615 times lesser risk of developing procedure-related injuries.

\section{DISCUSSION}

Endoscopy-related technological advances have brought both constructive and destructive prospects to the science and medical fields. According to the data shared by the American Society for Gastrointestinal Endoscopy, $43 \%$ of the work routine of gastroenterologists is usually spent performing endoscopy. ${ }^{12}$ Endoscopic submucosal dissection, endoscopic papillary balloon dilatation, and endoscopic sphincterotomy are among the few frequently selected and performed procedures with a prolonged duration, which increases the risk of ergonomic injuries among endoscopists. ${ }^{12}$ These procedural technologies ensure the effective treatment of gastrointestinal diseases with the aid of detailed imaging; however, they also enhance the overall risk of endoscopists for endoscopy-related injuries, MSK pain, and prolonged exposure to radiation. Therefore, to prevent these occupational injuries, ergonomic evaluation has become a necessity. Our findings also support the hypothesis that endoscopists are more likely to develop ergonomic injuries than non-endoscopists $(p<0.00)$ (Table 1), primarily owing to complex prolonged procedures and lack of necessary breaks. The overall prevalence of MSK injuries among endoscopists enrolled in the current study was $95.08 \%$, which is consistent with several other studies. ${ }^{9,10}$ Moreover, the sites of MSK pain varied among the two groups: pain in the back, leg, hand, and wrist was frequently reported among

Table 3. Factors Contributing to Ergonomic Injuries and Musculoskeletal Pain among Endoscopists

\begin{tabular}{lccc}
\hline Variable & OR $(\mathbf{9 5} \% \mathbf{C I})$ & p-value & Univariate $\boldsymbol{p}$-value \\
\hline Age $(<50$ yr vs. $>50$ yr $)$ & $1.008(0.86-1.17)$ & 0.542 & 0.920 \\
Endoscopies performed/week $(<20$ vs. $\geq 20)$ & $1.082(0.95-1.23)$ & 0.718 & 0.226 \\
Cumulative years $(<20$ yr vs. $\geq 20$ yr $)$ & $1.031(0.84-1.26)$ & 0.344 & 0.769 \\
Time spent performing endoscopy $(<5$ hr/wk vs. $>5$ hr/wk) & $0.465(0.12-1.71)$ & 0.490 & 0.250 \\
Regular breaks between procedure (yes vs. no) & $0.615(0.53-7.171)$ & 0.696 & 0.838 \\
\hline
\end{tabular}

$\mathrm{CI}$, confidence interval; OR, odds ratio.

${ }^{*} p$-value $<0.05$ is considered significant. 
endoscopists, whereas pain in the thumb, shoulder, and neck was common among non-endoscopists. Similarly, a study conducted by Ridtitid et al. to assess the prevalence of MSK injuries among endoscopists also showed similar results, in which upper back pain was most frequently reported among endoscopists followed by thumb, low back, and hand pain. ${ }^{12}$ The same findings were reported by Villa et al., who observed that the common sites of injuries were the right wrist, left thumb, back, and neck. ${ }^{16}$

Several factors influence endoscopy-associated MSK pain. Prolonged, repetitive use or overuse of integral parts of the body during endoscopic procedures are the major influencing factors. Adjusting tip angulation controls and torquing during a colonoscopy are among the endoscope-associated maneuvers that lead to endoscopy-specific injuries, specifically colonoscopist's thumb or de Quervain's tenosynovitis. Evidently, frequent high-volume prolonged procedures performed per week are the leading causes of overuse injury due to repetitive movements among endoscopists. ${ }^{12}$ Previous studies have suggested that performing endoscopic procedures for $>16 \mathrm{hr} /$ week (or 20 procedures) increases the risk of MSK injuries. ${ }^{10,12}$ Despite knowing the risks, endoscopists worldwide frequently exceed this threshold. A survey-based study also showed that surgeons performing $>30$ colonoscopies per week are at a higher risk of developing MSK injuries than those who perform $<30$ colonoscopies per week. ${ }^{2}$ In contrast, we found a higher incidence of MSK pain among endoscopists performing $<20$ procedures/week than among those performing $>20$ procedures/week. A possible explanation for this result may be that the presence of MSK pain causes these endoscopists to perform fewer procedures per week. Moreover, endoscopists performing $>20$ procedures/week were only 1.08 times more likely to have ergonomic injuries and MSK pain than those performing $<20$ procedures/week, indicating a non-significant difference with respect to procedural frequency. In addition to procedural frequency, improper positioning and prolonged therapies are other important factors leading to MSK injuries among endoscopists. ${ }^{9,10}$

Although working shifts are regulated to ensure the provision of quality health-care services by the HCPs, no practical procedural limitations exist for endoscopists. Moreover, with the extensive increase in the demand for endoscopic procedures, the number of procedures performed is also increasing. ${ }^{17}$ Therefore, ergonomic injuries are twice as frequent among endoscopists as compared with non-endoscopists owing to increased workload. ${ }^{16}$ All such influencing factors can be modified by taking mini-breaks between procedures, making postural improvements, exercising, decreasing the work duration, and decreasing the frequency of procedures per- formed per week. ${ }^{1}$ Surprisingly, most of the endoscopists with gastroenterology training (96.72\%) reported MSK injuries and pain. In contrast, a previous study reported very limited MSK injuries among HCPs with ergonomic training as compared with untrained endoscopists. ${ }^{16}$ The reason for the variation may be an inadequate training period or handling errors by endoscopists. $^{17}$

The lack of ergonomic education evidently plays an important role in decreased practice compliance and increased ratio of injuries among HCPs performing endoscopy. ${ }^{17}$ The incidence rate of ergonomic injuries could be minimized through the incorporation of ergonomic principles and techniques in daily practice. Endoscopists must be trained to maintain a net neutral body position throughout the procedure, to regulate the force and energy exertion without causing strain and injuries. ${ }^{18}$ The back and neck are the most common pain sites reported by the endoscopists, and one of the main reasons might be height-adjustment issues concerning the monitor or procedure table. ${ }^{18}$ The procedural setting must be well established and adjustable according to the requirements of the endoscopist, to ease painful and prolonged performance. ${ }^{19}$ Minirest periods and procedural breaks are necessary to prevent overuse injuries, as they effectively facilitate the rapid recovery of fatigued muscles. ${ }^{18}$ Moreover, wireless medical devices must be used for monitoring purposes because they reduce the chances of accidental falls in the endoscopy unit. ${ }^{9}$ These preventive measures might reduce, but do not eliminate, the risk of endoscopy-related injuries and pain.

The issue is worth addressing for better clinical practice of ergonomics and better outcomes not only for qualified but also skilled HCPs (endoscopists). ${ }^{20-22}$ Although our findings indicate a higher prevalence of ergonomic injuries among endoscopists than among non-endoscopists, several study limitations need to be considered. Among the studied predictors or contributing factors, none was found to significantly contribute to ergonomic injuries and MSK pain among endoscopists. One of the major reasons might be that most of the enrolled endoscopists had an MSK injury $(n=58)$, whereas only three had none. Therefore, the outcomes did not significantly predict the effect. Although several international studies with large sample sizes have been published on the prevalence of MSK injuries among endoscopists, ${ }^{1,5,12}$ large-scale, local descriptive studies assessing the prevalence of ergonomic injury-associated pain and its impact on occupational performance are required. Ergonomic assessment is necessary to prevent disabilities that can influence the efficiency and quality of life of endoscopists. Despite these limitations, our study explored the comparative prevalence of procedural injuries and MSK pain among endoscopists and non-endoscopists, and 
their preferred treatment choices. Moreover, it is one of the very few locally conducted studies involving both endoscopists and non-endoscopists, indicating a difference in the workplace environment, challenges, and risks of developing ergonomic injuries between the two groups.

In conclusion, ergonomic injuries are highly prevalent among endoscopists. Increased procedural frequency and prolonged procedural duration are more likely to cause endoscopy-related injuries. Further studies are required to investigate interventional alterations and modifications to prevent these injuries and ease the healing process among endoscopists. The ergonomic evaluation should be focused on the establishment of an adjustable procedural environment to prevent short- and long-term disabilities that can compromise the occupational performance and quality of life of endoscopists.

Conflicts of Interest

The authors have no potential conflicts of interest.

Funding

None.

\section{Acknowledgments}

The authors thank the Medical Affairs Department of Getz Pharma for assistance in statistical analysis and manuscript editing.

$\begin{array}{ll}\text { ORCID } & \\ \text { Lubna Kamani: } & \text { https://orcid.org/0000-0003-2651-5179 } \\ \text { Hamid Kalwar: } & \text { https://orcid.org/0000-0002-6937-6044 }\end{array}$

\section{REFERENCES}

1. O'Sullivan S, Bridge G, Ponich T. Musculoskeletal injuries among ERCP endoscopists in Canada. Can J Gastroenterol 2002;16:369-374.

2. Liberman AS, Shrier I, Gordon PH. Injuries sustained by colorectal surgeons performing colonoscopy. Surg Endosc 2005;19:1606-1609.

3. Byun YH, Lee JH, Park MK, et al. Procedure-related musculoskeletal symptoms in gastrointestinal endoscopists in Korea. World J Gastroenterol 2008; 14:4359-4364.

4. Hansel SL, Crowell MD, Pardi DS, Bouras EP, DiBaise JK. Prevalence and impact of musculoskeletal injury among endoscopists: a controlled pilot study. J Clin Gastroenterol 2009;43:399-404.

5. Kuwabara T, Urabe Y, Hiyama T, et al. Prevalence and impact of musculoskeletal pain in Japanese gastrointestinal endoscopists: a controlled study. World J Gastroenterol 2011;17:1488-1493.

6. Geraghty J, George R, Babbs C. A questionnaire study assessing overuse injuries in United Kingdom endoscopists and any effect from the introduction of the National Bowel Cancer Screening Program on these injuries. Gastrointest Endosc 2011;73:1069-1070.

7. Harris-Adamson C, Eisen EA, Kapellusch J, et al. Biomechanical risk factors for carpal tunnel syndrome: a pooled study of 2474 workers. Occup Environ Med 2015;72:33-41.

8. Shergill AK, McQuaid KR. Ergonomic endoscopy: an oxymoron or realistic goal? Gastrointest Endosc 2019;90:966-970.

9. Harvin G. Review of musculoskeletal injuries and prevention in the endoscopy practitioner. J Clin Gastroenterol 2014;48:590-594.

10. Singla M, Kwok RM, Deriban G, Young PE. Training the endo-athlete: an update in ergonomics in endoscopy. Clin Gastroenterol Hepatol 2018;16:1003-1006.

11. Dolay K, Hasbahçeci M. The role of surgeons on the development and performance of endoscopy. Turk J Surg 2017;33:1-4.

12. Ridtitid W, Coté GA, Leung W, et al. Prevalence and risk factors for musculoskeletal injuries related to endoscopy. Gastrointest Endosc 2015;81:294-302.e4.

13. Centers for Disease Control and Prevention. Work-related musculoskeletal disorders \& ergonomics [Internet]. Atlanta (GA): CDC; c2020 [updated 2020 Feb 12]. Available from: https://www.cdc.gov/workplacehealthpromotion/ health-strategies/musculoskeletal-disorders/index.html.

14. Lötters F, Burdorf A. Prognostic factors for duration of sickness absence due to musculoskeletal disorders. Clin J Pain 2006;22:212-221.

15. Centre for Workforce Intelligence. Securing the future workforce supply: gastrointestinal endoscopy workforce review [Internet]. London: CFWI; c2017 [updated 2017 Mar]. Available from: https://assets.publishing. service.gov.uk/government/uploads/system/uploads/attachment_data/ file/597718/Gastrointestinal_endoscopy_workforce_review.pdf.

16. Villa E, Attar B, Trick W, Kotwal V. Endoscopy-related musculoskeletal injuries in gastroenterology fellows. Endosc Int Open 2019;7:E808-E812.

17. Lipp MJ. The risks of GI endoscopy. Gastrointest Endosc 2009;69:11491151.

18. Eastman Kodak Company. Kodak's ergonomic design for people at work. 2nd ed. Hoboken (NJ): Wiley; 2004.

19. ASGE Quality Assurance In Endoscopy Committee; Petersen BT, Chennat J, et al. Multisociety guideline on reprocessing flexible gastrointestinal endoscopes: 2011. Gastrointest Endosc 2011;73:1075-1084.

20. Edelman KM, Zheng J, Erdmann A, et al. Endoscopy-related musculoskeletal injury in AGA gastroenterologists is common while training in ergonomics is rare. Gastroenterology 2017;152(5 Suppl 1):S217.

21. Chang MA, Mitchell J, Abbas Fehmi SM. Optimizing ergonomics before endoscopy. VideoGIE 2017;2:169.

22. Chang MA, Mitchell J, Abbas Fehmi SM. Optimizing ergonomics during endoscopy. VideoGIE 2017;2:170. 